\title{
Biomarkers for primary biliary cholangitis: current perspectives
}

This article was published in the following Dove Press journal: Hepatic Medicine: Evidence and Research

\author{
Elias Kouroumalis' \\ Demetrius Samonakis ${ }^{2}$ \\ Argyro Voumvouraki ${ }^{3}$ \\ 'Department of Gastroenterology, \\ University Hospital and Medical \\ School, University of Crete, Heraklion, \\ Crete, Greece; ${ }^{2}$ Department of \\ Gastroenterology, University \\ Hospital of Heraklion, Crete, Greece; \\ ${ }^{3}$ Department of Medicine, AHEPA \\ University Hospital, Thessaloniki, \\ Greece
}

\begin{abstract}
Primary biliary cholangitis (PBC) is a chronic progressive cholestatic disease characterized by destruction of small- and medium-sized intrahepatic bile ducts. It is no longer a rare disease, since many new asymptomatic cases are incidentally identified. Liver biopsy is diagnostically critical but not always feasible or practical to be performed. Many potential, noninvasive, markers have been proposed to replace liver biopsy and further provide the assessment of disease severity and ultimate prognosis. In this review, we evaluated serum biomarkers proposed for diagnosis, extent of fibrosis, disease prognosis and attempts for early prediction of treatment response. Older biochemical and immunological markers are presented along with recent reports including the role of microRNAs and promising results based on proteomics and metabolomics.
\end{abstract}

Keywords: primary biliary cholangitis, autoantibodies, microRNAs, proteomics, treatment response

\section{Introduction}

Primary biliary cholangitis (PBC) is an autoimmune cholestatic disease of unknown etiology with a varying geographical incidence. Prevalence ranges between 1.91 and 40.2 per 100,000 population. ${ }^{1,2}$

According to the American Association for the Study of Liver Diseases, the diagnosis of PBC is certain when two of the following three criteria are met: 1) biochemical evidence of cholestasis based mainly on alkaline phosphatase (ALP) elevation; 2) the presence of anti-mitochondrial antibodies (AMAs) and 3) histological evidence of intrahepatic destructive cholangitis of interlobular bile ducts. AMAs are recognized as the gold biomarkers. ${ }^{3}$

However, there are problems with these criteria in clinical practice. There is a PBC patient subpopulation where AMAs are negative even with the most sensitive methods. These patients require a liver biopsy for diagnosis which may be problematic when ascites or infections are present. It is not easily accepted by the patient although the complication rate is low when performed under ultrasound guidance. A need therefore exists for easily applicable noninvasive biomarkers that could be used in diagnosis, prognosis, fibrosis assessment and treatment prediction.

The current review will therefore evaluate the existing knowledge of serum biomarkers in patients with PBC.
Correspondence: Elias Kouroumalis Department of Gastroenterology, University Hospital of Heraklion, 7I 100 Heraklion, Crete, Greece

Tel +302810392356

Fax +302810542085

Email kouroumi@uoc.gr 


\section{Biomarkers for PBC diagnosis Immunological markers}

Immunological biomarkers were a fundamental element in the diagnosis of $\mathrm{PBC}$ ever since the initial clarification of the disease (Table 1). Even today, the presence of AMAs is among the three major diagnostic criteria. ${ }^{3}$

AMAs are found in more than $90 \%$ of patients with PBC with less than $1 \%$ prevalence in the general population, and they are not related to disease progression. ${ }^{4,5}$ AMA levels do not significantly change over time or after treatment irrespective of response. ${ }^{6}$

AMAs are in fact a family of immunologically very similar antibodies. Nine subtypes have been described, but only four are closely associated with PBC: AMA-M2, AMA-M4, AMA-M8 and AMA-M9. AMA-M2 is the subtype mostly used as a routine diagnostic marker of PBC. ${ }^{7}$

The target antigen was identified as the inner lipoyl domain of the E2 subunit of pyruvate dehydrogenase complex. Further analysis revealed the necessity of the lipoyl $\beta$-sheet structural conformation in anti-PDC-E2 recognition. ${ }^{8}$

The sensitivity and specificity of AMAs in PBC depend on the method used. Most routine laboratories use indirect immunofluorescence (IIF-AMA) with a cutoff titer of 1:40 and a sensitivity and specificity around $88.0 \%$ and $97.0 \%$, respectively, but a higher cutoff titer of 1:80 is suggested to be used for the maximum benefit to be obtained. ${ }^{9}$ Four commercial assay kits for AMA-M2 autoantibodies were also tested with a sensitivity of $55.7-79.7 \%$ and specificity of 91.7-95.4\%. AMA-M2 assays with both native and recombinant $\mathrm{E} 2$ antigens were characterized by better sensitivity. ${ }^{10}$

An interesting recent report offered patients a more convenient diagnosis. Saliva was tested for the presence of AMA-M2 and was found positive only when serum was also positive. The saliva concentration significantly correlated with serum AMA-M2. The area under the curve (AUC) was 0.88 with a sensitivity of $81.82 \%$ and specificity of $80.00 \% .^{11}$

The AMA-negative cases are an intriguing diagnostic problem. The actual prevalence of this entity varies and most probably depends on the expertise of the laboratory and the method used. Enzyme-linked immunosorbent assay (ELISA) has a sensitivity and specificity higher than IIF. The three E2 subunits included in the MIT3 assay are: the E2 subunit of the puryvate dehydrogenase complex-PDC-E2, the branchedchain 2-oxo-acid dehydrogenase complex-BCOADC-E2, and the 2-oxo glutarate dehydrogenase complex -OGDC-E2. MIT3 ELISA could detect the presence of AMA in half of the AMA-negative sera by IIF. ${ }^{12}$ A more sensitive ELISA using cloned antigens from human sources increased the sensitivity to $94 \%$ (compared to $84 \%$ by IIF), but most importantly $73 \%$ of PBC patients with negative AMA by IIF were tested positive. $^{13}$

A critical review of methods for the detection of AMA has recently been published. ${ }^{14}$

A subgroup of PBC patients does not have AMA antibodies, but they are clinically and histologically identical to classical PBC. Immunological biomarkers apart from AMAs are particularly useful for this group of patients. These include various antinuclear antibodies (ANAs) detected by immunofluorescence. Two distinct patterns are strongly associated with PBC: the multiple nuclear dot and rim-like/membranous patterns. The target antigens of the multiple nuclear dot patterns are the sp100 protein and the PML protein. The rimlike/membranous pattern represents antibodies targeting the nuclear pore complex antigen gp210, the nucleoporin antigen p62 and the lamin B receptor. ${ }^{15,16}$ gp210 and sp100 are the ANAs most extensively investigated with very high specificity. Anti-centromere antibodies are also ANAs frequently found in PBC patients but are not specific. ${ }^{17}$

Detection rates of anti-gp210 vary in different geographical areas. Thus, a report on Spanish and Greek patients reported only $10.6 \%$ positivity for gp $210 .{ }^{18}$ However, our study in Greek patients found a $21 \%$ positivity for gp $210,{ }^{19}$ a similar percentage with Japanese patients $(26 \%) .{ }^{20}$ A metaanalysis of 25 studies on gp 210 and 21 studies on sp 100 with almost 13,000 PBC patients from different countries reported a very high specificity ( 0.985 for gp210 and 0.977 for sp100), but low sensitivity for the diagnosis of PBC ( 0.272 and 0.231 for gp210 and sp100, respectively). ${ }^{21}$

An ELISA screening test including two ANAs (gp210, sp100) and a triple (pMIT3) AMA recombinant antigen showed that $43 \%$ of patients previously diagnosed as having AMA-negative $\mathrm{PBC}$ were tested positive and therefore a liver biopsy was not necessary for diagnosis. ${ }^{22}$

Other ANAs include the anti-p62 antibodies that react with a $60 \mathrm{kD}$ antigen of the nuclear pore complex with a specificity of almost $97 \%$. However, sensitivity is again very low. ${ }^{23}$ Detection of antibodies to PML protein does not seem to offer an extra diagnostic tool as only $25-30 \%$ of AMA-positive and AMA-negative patients are positive. ${ }^{24}$ The same is true for antibodies against a new PML nuclear body protein, designated as sp140. Anti-sp140 was always detected in parallel with anti-sp100 irrespective of AMA status. ${ }^{25}$

A new multiplexed line-blot assay, namely ALD2, has been tested in a small series of PBC patients. Antigens incorporated were: AMA-M2, a recombinant fusion protein 
Table I Main studies of biomarkers in PBC

\begin{tabular}{|c|c|c|c|c|c|}
\hline Diagnosis & Biomarker & $\begin{array}{l}\text { No. of patients } \\
\text { and studies }\end{array}$ & Sensitivity (\%) & Specificity (\%) & AUC \\
\hline \multirow[t]{2}{*}{ Hua et $\mathrm{al}^{9}$} & AMA/M2 & 24 studies & 84.5 & 98 & 0.97 \\
\hline & & Meta-analysis & & & \\
\hline \multirow[t]{3}{*}{ Han et $a^{10}$} & AMA IF I/40 & 79 & 88.6 & 87 & \\
\hline & IF I/80 & & 80 & 93.5 & \\
\hline & 4 commercial tests & & 83.5 & 90.7 & \\
\hline \multirow[t]{2}{*}{ Lu et al" } & M2 saliva & 49 & 81.8 & 80 & 0.88 \\
\hline & Only in AMA + patients & & & & \\
\hline \multirow[t]{3}{*}{ Hua et al ${ }^{9}$} & ANA gp210 & 25 studies & 27 & 98.5 & 0.53 \\
\hline & spl00 & 21 studies & 23 & 97.7 & 0.32 \\
\hline & & 13,000 patients & & & \\
\hline \multirow[t]{3}{*}{ de Liso et $\mathrm{a}^{30}$} & gp210 & Eight studies & 38 & 90 & \\
\hline & spl00 & AMA-negative & 42 & 91 & \\
\hline & Anti-KLHLI/HKI & patients & 25 & $86-96$ & \\
\hline \multirow[t]{2}{*}{ Hao et $\mathrm{al}^{34}$} & Metabolomics & 51 & 80 & 90 & 0.93 \\
\hline & Glucose, fatty acids, amino acids & & & & \\
\hline \multirow[t]{3}{*}{ Tan et $\mathrm{a}^{43}$} & miRNAs & 82 & 80.5 & 88 & 0.905 \\
\hline & miR-122-5p & & Increased & & \\
\hline & miR-|4|-3p & & & & \\
\hline \multirow[t]{2}{*}{ Katsumi et $\mathrm{al}^{42}$} & miR-139-5p & 45 & Decreased in advanced & & \\
\hline & & 45 & disease & & \\
\hline Prognosis & & & Hazard ratio & & \\
\hline Wesierska-Gadek et al ${ }^{60}$ & Anti-NCP (gp2।0) & 127 & 3.4 & & \\
\hline \multirow[t]{2}{*}{ Miyachi et $a^{64}$} & Anti-p62 & 175 & $27 \%$ confirmation of & & \\
\hline & & & Scheuer stage IV & & \\
\hline \multirow[t]{2}{*}{ Nakamura et al ${ }^{|6|}$} & gp210 & 71 & End-stage liver failure $60 \%$ & & \\
\hline & & & when high & & \\
\hline Nakamura et a ${ }^{20}$ & gp210 & 276 & 6.74 & & \\
\hline Fibrosis & Combination biomarkers & $\begin{array}{l}\text { No. patients and } \\
\text { studies }\end{array}$ & & & \\
\hline \multirow[t]{2}{*}{ Xie et al96 } & ELF & Nine studies & 83 & 73 & 0.88 \\
\hline & & Meta-analysis & & & $\begin{array}{l}\text { Severe fibrosis- } \\
\text { cirrhosis }\end{array}$ \\
\hline \multirow[t]{4}{*}{ Wang et al97 } & RDW & 73 & 33.3 & 92.9 & 0.66 \\
\hline & RPR & Scheuer stage >II & 46.7 & 96.4 & 0.74 \\
\hline & APRI & & 88.9 & 39.3 & 0.65 \\
\hline & FIB-4 & & 57.8 & 78.6 & 0.68 \\
\hline \multirow[t]{5}{*}{ Wang et al98 } & APRI & 58 & 76.5 & 73.2 & 0.726 \\
\hline & FIB-4 & Scheuer stage $>$ II & & & 0.722 \\
\hline & MPV & & & & $0.67 I$ \\
\hline & RPR & & & & 0.717 \\
\hline & PL/ST & & & & 0.807 \\
\hline \multirow{10}{*}{$\begin{array}{l}\text { Prediction models of } \\
\text { treatment response }\end{array}$} & Model & Criteria & No. of patients & Assessment & \\
\hline & & & & $\begin{array}{l}\text { after induction } \\
\text { of therapy }\end{array}$ & \\
\hline & Barcelona ${ }^{105}$ & $\begin{array}{l}\text { ALP: normalization } \\
\text { or }>40 \% \text { reduction }\end{array}$ & 192 & I year & \\
\hline & Paris-I 106 & $A L P<3.0 \times U L N$ & 292 & I year & \\
\hline & & AST $<2.0 \times U L N$ & & & \\
\hline & & Bilirubin $<$ I mg/dL & & & \\
\hline & Toronto ${ }^{107}$ & $A L P<1.67 \times \cup L N$ & 69 & 2 years & \\
\hline & Paris-II ${ }^{108}$ & $\mathrm{ALP}<\mathrm{I} .5 \times \mathrm{ULN}$ & 165 & I year & \\
\hline & & AST $<1.5 \times U L N$ & & & \\
\hline & & Bilirubin $<1 \mathrm{mg} / \mathrm{dL}$ & & & \\
\hline
\end{tabular}

Abbreviations: ALP, alkaline phosphatase; AMA, anti-mitochondrial antibody; ANA, antinuclear antibody; APRI, AST/platelet ratio index; AST, aspartate aminotransferase; AUC, area under the curve; ELF, enhanced liver fibrosis; FIB-4, fibrosis index based on four factors; HKI, hexokinase-I; KLHL7, Kelch-like protein 7; microRNAs, miRNAs; PBC, primary biliary cholangitis; RDW, red blood cell distribution width; RPR, RDW/platelet ratio; ST, spleen thickness; IF, immunofluorescence; MPV, mean platelet volume; PL, platelets; ULN, upper limit of normal. 
including the E2 subunits of PDC, BCOADC and OGDC (M2-E3), sp100, PML and gp210 recombinant proteins. Excellent overall sensitivity and specificity of $98.3 \%$ and $93.7 \%$, respectively, were reported, but the test should be validated in larger series. ${ }^{26}$

\section{Proteomics}

Progress in diagnostic biomarkers might come from contemporary analytical techniques. Thus, proteomics, the analytical identification of the role of proteins, have provided encouraging results.

The use of MALDI-TOF-MS (Ciphergen Biosystems, USA) combined with magnetic beads and pattern recognition software identified 69 discriminant $\mathrm{m} / \mathrm{z}$ peaks associated with PBC. The $\mathrm{m} / \mathrm{z}$ peaks at $3445,4260,8133$ and 16,290 were used to construct a diagnostic model for PBC with an excellent sensitivity of $93.3 \%$ and a specificity of $95.1 \%$. This model needs further extensive validation studies in different PBC subgroups. ${ }^{27}$

Using proteomic techniques, two new PBC-associated antigens namely hexokinase-1 (HK1) and Kelch-like protein 7 (KLHL7) were detected. Anti-KLHL12 and anti-HK1 antibodies were detected in 10-35\% of AMA-negative patients, and when combined with gp210 and sp100, they increased the sensitivity in AMA-negative patients from 55\% to $75 \%{ }^{28-30}$

\section{Metabolomics}

Use of metabolomic techniques may in future shift the diagnostic tools from autoantibodies to metabolites.

Thus, 420 serum metabolites from PBC patients and controls were identified. A total of 101 of them significantly differed between $\mathrm{PBC}$ patients and controls and 56 significantly differed between primary sclerosing cholangitis and PBC. The significance in clinical diagnosis should be further investigated. ${ }^{31}$

Bile acid and lipid metabolic products can also be used for diagnostic purposes in PBC. In findings observed in a study conducted in China, the serum concentrations of bile acids were increased in PBC, while the concentrations of carnitines were reduced. Carnitines may be of value as PBC biomarkers for both diagnosis and outcome prediction. ${ }^{32}$ Another study reported that bile acids were increased while metabolites associated with lipid metabolism were decreased in patients compared to controls. Although these findings are still preliminary, they might lead to the creation of diagnostic algorithms. ${ }^{33}$

In the most complete study so far, training and validation groups of PBC patients were used to assess metabolomic biomarkers related to glucose, fatty acid and amino acid metabolites. In receiver operating characteristic (ROC) analysis, an impressive AUC of 0.937 for the training group was reported accompanied by an equally impressive 0.890 for the validation group making metabolomics a feasible diagnostic tool. ${ }^{34}$

Comprehensive reports have recently been published. ${ }^{35,36}$

\section{microRNAs (miRNAs): possible future biomarkers in PBC}

Additional information on the evolution of PBC might emerge from the study of miRNAs.

They are small noncoding RNAs that regulate almost $60 \%$ of gene expression. They have been proposed as useful biomarkers in several diseases including $\mathrm{PBC}^{37}$ to assist in diagnosis and prognosis.

As diagnostic tools, 35 differentially expressed miRNAs were identified (11 upregulated and 24 downregulated) in liver tissues from $\mathrm{PBC}$ patients compared to tissues from normal controls. ${ }^{38}$

Different miRNA profiles were also found in peripheral blood mononuclear cells (PBMCs) of PBC patients compared to healthy controls, ${ }^{39}$ while the miRNA-let- $7 \mathrm{~b}$ was downregulated and more importantly correlated with disease severity. ${ }^{40}$

In a small group of 10 PBC patients, a unique mRNA expression pattern was identified. Downregulation of hsamiR-505-3p, hsa-miR-139-5p and hsa-miR-197-3p was proposed as a useful diagnostic tool. Validation is obviously required. ${ }^{41}$ Further investigation from the same group showed that the liver expression of 97 miRNAs significantly varied in the clinical subtypes of PBC. miRNA profiles were different in the slowly progressive type compared to the hepatic failure and portal hypertension types. Again miR-139-5p was downregulated in clinically advanced PBC. ${ }^{42}$

A different set of miRNAs (hsa-miR-122-5p, hsa-miR141-3p, hsa-miR-26b-5p) was reported in another study. It has a very high diagnostic accuracy for PBC with an AUC of 0.905 , but most importantly with an encouraging high sensitivity of $80.5 \%$ accompanied by an equally high specificity of $88.3 \%{ }^{43}$

A total of 16 miRNAs were also differentially expressed in PBC compared to healthy controls. The most prominent finding was the downregulation of miR-92a. Interestingly, the expression of miR-92a was colocalized with interleukin (IL)-17A in PBMCs of patients, implying a direct regulation of IL-17A by miR-92a, thus connecting miRNA-92a to disease progression. ${ }^{44}$ In that respect, it should be noted that there is a direct association of Th17 infiltration with disease severity implicating the IL-23/Th17 lymphocytes pathway in the immunopathology in PBC. ${ }^{45}$ In addition, serum levels of 
IL-23 and IL-17 in PBC patients were increased compared to controls and positively correlated with serum $\gamma \mathrm{GT}$. There was also a correlation with clinical stages making them useful indices to monitor PBC. ${ }^{46}$

Downregulation of the $\mathrm{Cl}^{-} / \mathrm{HCO}_{3}{ }^{-}$anion exchanger 2 (AE-2) and the type III inositol 1,4,5-trisphosphate receptor (InsP3R3) of cholangiocytes is implicated in the pathogenesis of cholestatic syndromes including PBC. miR-506 is one of the triggering causes for both AE-2 and InsP3R3 downregulation in PBC cholangiocytes. ${ }^{47,48}$ Further research from the same group identified various pro-inflammatory cytokines, overexpressed in PBC liver that stimulated miR-506 expression in human cholangiocytes inducing PBC-like features and immune activation. ${ }^{49}$ In connection with these findings, it is interesting that a recent report demonstrated that downregulation of miR-425 in CD4+ T cells from PBC patients induced overproduction of pro-inflammatory cytokines possibly indicating a step further up the immunopathological pathway of PBC. ${ }^{50}$

miRNA deregulation in peripheral $B$ cells of $P B C$ patients from stage I to stage IV was recently investigated. Although more than 500 miRNAs were differentially expressed in patients, only hsa-miR-223-3p and hsa-miR-21-5p showed consistent downregulation from stage I to stage III suggesting that they are related to disease progression. ${ }^{51}$

Extracellular vesicles (EVs) are nano- or micro-lipid bilayer spheres produced by different cells. They are released into the extracellular space where they participate in intercellular communications. They are also found in bile and contain miRNAs. ${ }^{52}$ In PBC, plasma EVs regulate the expression of the co-stimulatory molecules such as CD86 and CD80 in peripheral antigen-presenting cells. PBC plasma-derived EVs had increased quantities of miR-451a and miR-642a-3p with immunomodulatory functions compared to healthy controls. ${ }^{53}$

Three informative reviews on the subject have recently been published, ${ }^{54-56}$ but the promising results require further validation.

In conclusion, the diagnosis of AMA-positive PBC can entirely be based on biomarkers. The most difficult diagnostic problem is the AMA-negative PBC, where a central diagnostic element is missing even if the most sensitive techniques are employed. A recent meta-analysis of eight selected reports from AMA-negative patients exemplifies the current situation. All the available immunomarkers were included. A pooled sensitivity and specificity of $37 \%$ and $85 \%$, respectively, were calculated. The overall positive predicted value (PPV) and negative predicted value (NPV) respectively, were $45 \%$ and $83 \%$. Interestingly, this meta-analysis included the recently described novel autoantigens such as KLHL12 and HK1. Although specificity of each individual marker is very high, the individual and overall sensitivity are poor. ${ }^{30}$

It is therefore highly recommended that in suspected PBC cases without AMAs, the detection of gp210, sp100 and ideally anti-KLHL12/HK1 should be the next step. The new multiplexed line-blot assay ALD2 should preferably be used if initial promising results are verified. ${ }^{26}$ Although a positive result practically confirms the diagnosis, a negative result does not exclude the diagnosis and a liver biopsy remains mandatory.

\section{Markers for prognosis Immunological markers}

$\mathrm{PBC}$ has two fundamental characteristics. First, the natural course may be extremely slow. The disease usually, but not always, evolves over decades rather than years. Second, its clinical presentation is not uniform. Three clinical types are recognized. The slow progression asymptomatic type, the liver failure with jaundice and hepatic encephalopathy type and the portal hypertension with variceal bleeding type. ${ }^{20,42}$ Therefore, a stratification is critical but unfortunately is not usually attempted in prognostic studies.

Various biochemical and immunological markers have been used to predict disease progress. Although AMAs are not associated with prognosis, earlier reports have focused on the prognostic significance of AMA isotypes. Patients with only anti-M4 seem to have slower disease progression and benign outcome, whereas patients having anti-M2, anti-M4 and anti-M8 seem to have more active disease and worse outcome. ${ }^{57}$ This was later disputed in a study from the Netherlands. ${ }^{58}$ The presence of the IgG3 isotype of AMA has been related to a more severe disease. ${ }^{59}$

Other PBC-specific antibodies, particularly ANAs, are more promising as prognostic markers. Specific types of ANAs, such as the anti-gp210, and anti-centromere antibodies, have been associated with a worse prognosis. ${ }^{60,61}$

In a Japanese study, two clinical subgroups of patients were identified, one dying of hepatic failure and the other developing esophageal varices and hepatocellular carcinoma without jaundice (portal hypertension type). Anti-gp210 antibodies were a risk factor for hepatic failure (odds ratio 33.77), while anti-centromere antibodies were a risk factor for the portal hypertension type (odds ratio 4.20). ${ }^{20}$ This was not verified in a group of Spanish and Greek patients. ${ }^{18}$ However, in our study of antinuclear envelope antibodies (ANEAs) in Greek patients, positivity was associated with a more advanced histological stage and a lower survival time. 
According to the Japanese study, ANEA-positive patients died more frequently of hepatic failure, while ANEA-negative patients died more frequently of variceal bleeding. It should be noted that we used a slight modification of IIF, with a $1 \%$ fixation instead of $4 \%$ that allowed for much better visualization of peri-nuclear staining. ${ }^{19}$ An interesting investigation found that anti-centromere antibodies are related to changes of estimated glomerular filtration rate (eGFR) in PBC patients and that they are an independent predictor of the development of chronic kidney disease. ${ }^{62}$

The significance of anti-centromere antibodies has been recently reviewed. ${ }^{63}$

Anti-p62 antibodies have also been associated with worse prognosis. A multicenter study showed that anti-p62 antibodies are related to advanced stage of $\mathrm{PBC}$, and their prevalence is higher in symptomatic patients with cirrhosis or its complications. ${ }^{64}$

Most autoantibodies do not significantly change over time and changes do not correlate with clinical progression in PBC with a possible exception of sp100. The sp100 slope over time was inversely associated with fibrosis. ${ }^{65}$

\section{Markers without proper validation}

Many other biomarkers have been used as prognostic factors in PBC. Most of them have not been properly validated and their use is still under investigation. The intermediate filaments of normal hepatocyte cytoskeleton primarily consist of keratins, K8 and K18 with a functional role in the mechanical stability of the hepatocyte. ${ }^{66}$ Apoptosis in liver disease leads to cleavage of $\mathrm{K} 18$ by caspase- 2 . K18 fragments are released into the circulation and can be measured by ELISA. ${ }^{67} \mathrm{~K} 18$ fragment levels were elevated in patients with $\mathrm{PBC}$ using assays that detect either M65 (antigen found in caspasecleaved and uncleaved K18, a marker of overall cell death from apoptosis or necrosis), or M30 (an exposed neo-epitope after cleavage of K18). ${ }^{68}$

The levels of M30 and M65 were significantly correlated with fibrosis and are different in fibrosis stages. Values from a newly developed M65ED ELISA were associated with poor clinical outcome at a cutoff value of $672 \mathrm{UI} / \mathrm{L} .{ }^{69}$

A biomarker predicting a favorable outcome has been identified. An antibody reacting with the p97/valosin-containing protein (VCP) was detected in a minority of PBC patients followed up for an average of 15 years with a very slow progress and no mortality. ${ }^{70}$

Autotaxin (ATX) is implicated in the synthesis of lysophosphatidic acid and has been incriminated in the pathogenesis of pruritus of cholestasis. ATX was significantly increased in PBC patients particularly those with cirrhosis but most importantly it was a negative predictor of survival. High levels were associated with a fourfold increased risk of death or transplantation. ${ }^{71}$

Fibroblast growth factor 19 (FGF19) is a regulatory molecule of liver bile acid synthesis which is deranged in PBC. Serum levels of FGF19 correlated with the severity of liver disease, worse liver biochemistry and worse quality of life. ${ }^{72}$

Serum total cholesterol and bile acids may predict liver failure in PBC..$^{73}$ Increased serum levels of the chemokine fractalkine could serve as a predictor of cholangitis activity at early stages ${ }^{74}$ Components of extracellular matrix have also been investigated. Thus, a serum cartilage oligomeric matrix protein has been tested as a novel biomarker of cirrhosis in various chronic liver diseases. It is higher in other cirrhotic liver disease compared to non-cirrhotics but is unable to differentiate between cirrhotic and non-cirrhotic PBC patients. ${ }^{75}$

Another component of extracellular matrix, vitronectin, was increased in both AMA-negative and AMA-positive PBC patients, and an association with serious bile duct destruction was established. ${ }^{76}$

Another protein, the Wisteria floribunda agglutininpositive Mac-2-binding protein [WFA (+)-M2BP], has been proposed as a very good predictor of clinical prognosis. ${ }^{.7}$

\section{Prognostic models}

Various prognostic models based on single biochemical parameters have been developed in an effort to predict clinical outcome in PBC. The aspartate aminotransferase (AST) to platelet ratio index (APRI) is one of the better validated models. Two recent reports have confirmed previous studies indicating that elevated APRI is a predictor of increased disease progression in PBC. ${ }^{78,79}$ APRI at 1 year (APRI-r1) was investigated as a progression predictor in Chinese patients followed up for 5, 10 and 15 years; APRI-r1 AUCs were satisfactory: 0.800 .83 , and 0.77 , respectively, making therefore APRI a useful tool in the clinical assessment of PBC patients in multiple ethnicities.$^{80}$ In a group of 204 patients, however, APRI was significantly correlated with the histological degree of liver fibrosis but the PPV was very low limiting its uniform applicability in clinical practice. ${ }^{81}$

It should be noted however that most models based on biochemical markers have been devised to assess the progression of liver fibrosis in $\mathrm{PBC}$ and only indirectly are predicting clinical outcome although one should keep in mind that fatal complications in PBC may develop before cirrhosis. ${ }^{82}$

In conclusion, the ideal marker for prognosis is still required probably due to the variable natural course of the 
disease. gp210 antibodies and anti-centromere antibodies are the most extensively validated biomarkers so far. It is hoped that one or more of the markers without proper validation so far will be the answer. It should be remembered however that the evolution of two old biochemical markers, namely ALP and bilirubin, are still among the best when prognosis is concerned. Data are presented in the Markers for treatment success section.

\section{Markers for fibrosis}

\section{Single biomarkers}

Among different proposed single biochemical markers, only serum hyaluronan seems to be the most promising biomarker for the assessment of fibrosis in PBC. The AUC of late PBC was 0.74 for hyaluronan, 0.63 for leptin, 0.59 for laminin and 0.70 for collagen IV. Hyaluronan also had high sensitivity and NPV in identifying late stages of PBC (96\% and 90\%, respectively). ${ }^{83}$ However, inconsistent results of hyaluronan, laminin, collagen IV and pro-collagen III in different Child-Pugh stages have been reported, possibly making these markers not suitable for clinical practice. ${ }^{84}$

Mean platelet volume and bilirubin were investigated in a small study, but AUC was only 0.72 with low specificity and sensitivity, which was certainly not better than hyaluronan. ${ }^{85}$

The WFA (+)-M2BP has also been used as a biomarker for discriminating the fibrotic stages in $\mathrm{PBC}^{78}$ and later verified in a study where the serum levels of chemokine IP-10 also gave similar results. ${ }^{86}$ In fact, both these biomarkers were only able to identify extensive fibrosis, but as another study has shown, there is considerable overlap with earlier fibrotic stages. ${ }^{87}$

A glycan isomer (M2BPGi) of this protein has been recently proposed as a reliable marker of liver fibrosis in $\mathrm{PBC}$, but it is too early to draw firm conclusions. ${ }^{88}$

\section{Models combining biomarkers}

To improve the situation over single biomarkers, several models have been proposed. The AST/alanine aminotransferase (ALT) ratio, the APRI score, the fibrosis index based on 4 factors (FIB-4), the Fibro index, the Forns and the enhanced liver fibrosis (ELF), among others, have been most extensively validated indicating that the ideal model does not exist. ${ }^{89}$

It was earlier suggested that the AST/ALT ratio was significantly higher in cirrhotic patients than in non-cirrhotic patients and was significantly associated with esophageal varices and ascites, ${ }^{90}$ but a more recent report found an AUC of only 0.660 for discriminating the fibrotic stages of $\mathrm{PBC}$, which was marginally better than APRI. ${ }^{11}$
One of the serious problems that all proposed models face is the relative inability to discriminate the four histological stages of PBC. This has been convincingly demonstrated in recent publications. ${ }^{92,93}$ The best that these models can do is to discriminate between early and advanced stages of PBC. Thus, a statistically significant difference in APRI, FIB-4 and AST levels between groups with early and advanced PBC has been reported. ${ }^{94}$ The ELF test, based on serum hyaluronan, tissue inhibitor of metalloproteinase 1 and procollagen III aminopeptide, was shown to have prognostic ability even in the early stages of the disease process (area under receiver operating characteristic $[$ AUROC $]=0.737-0.863$ at all time points)..$^{95}$ A recent meta-analysis of nine studies concluded that the ELF test had a pooled sensitivity of $83 \%$, specificity of $73 \%$ and AUC of 0.88 for significant or severe fibrosis and cirrhosis, but the sample included patients with other liver diseases as well. ${ }^{96}$

The red blood cell distribution width (RDW) and RDW to platelet ratio (RPR) were proposed as markers of the histologic severity of PBC. Again, there was a low sensitivity of $46.7 \%$ and a high specificity of $96.4 \%$ with an AUC of 0.74. Compared to APRI (AUC=0.648) and FIB-4 (0.682), RPR seems to be slightly better but only for discrimination between early and late disease. ${ }^{97}$ The same group very recently compared APRI, FIB-4, RPR and platelet count to spleen thickness (PC/ST) ratio. This new marker improved sensitivity to almost $75 \%$ for discrimination among the four Scheuer PBC stages. ${ }^{98}$

In conclusion, a biomarker is considered perfect if the AUROC is $100 \%$, excellent if the AUROC is $>90 \%$ and good if the AUROC is $>80 \% .{ }^{99}$ Currently, there are excellent noninvasive tests to exclude, but not to diagnose, extensive fibrosis or cirrhosis. ${ }^{100}$ Moreover, they are unable to discriminate between the different histological stages of PBC.

\section{Markers for treatment success}

Ursodeoxycholate (UDCA) was the only approved treatment of PBC up to 2016. Obeticholic acid is now approved in the USA and Europe as a second-line drug in patients after a UDCA failure. It should be noted however that a recent large meta-analysis of 46 trials concluded that there is currently no evidence that any intervention is beneficial for PBC. Overall, all the evidence was of low or very low quality, and the follow-up periods in the trials were rather short. Therefore, no firm conclusions can be drawn. ${ }^{101}$

Liver biopsy is the gold standard for the evaluation of treatment success, but it is not a practical clinical option. 
There have been many efforts to identify biomarkers to predict successful treatment.

A longitudinal study of 110 patients suggested that declining sp100 titers correlated with improvement in the Mayo risk score and response to UDCA. ${ }^{102}$

Vitamin D has been implicated in immune modulations. Vitamin D deficiency is highly prevalent in the PBC group with almost one-third of these patients exhibiting serum vitamin D levels below $10 \mathrm{ng} / \mathrm{mL} .{ }^{103}$

The vitamin D levels were decreasing as histological stages increased and were negatively correlated with bilirubin and ALP. A low baseline vitamin D level was associated with an increased risk of incomplete response independent of advanced stages. ${ }^{104}$

The levels of serum ALP are a significant prognostic factor and were extensively used in prediction models (Table 1). The first was the Barcelona prediction model, where an ALP normalization or a decrease $(>40 \%)$ from baseline after 1 year of UDCA was associated with a favorable response. ${ }^{105}$ The Paris-I then appeared with a $90 \%$ survival for those fulfilling the criteria compared to $51 \%$ for those with higher values. ${ }^{106}$ The Toronto prediction model was next ${ }^{107}$ followed by the Paris-II. ${ }^{108}$

However, a direct comparison of these models in early PBC indicated that criteria at 1 year of UDCA administration reflect the severity of the disease rather than the therapeutic response to UDCA. ${ }^{109}$

Findings on ALP were later verified by a large multinational study, where levels of ALP $<2$ upper limit of normal and bilirubin $<1 \mathrm{mg} / \mathrm{dL}$ were strong predictors of survival after UDCA. ${ }^{110}$ The same group devised a more complex prognostic score, namely GLOBE, which included age, bilirubin, albumin, ALP, and PC after 1 year of treatment. The GLOBE score predicted survival of UDCA responders at 5 and 10 years with PPV respectively. ${ }^{111}$

Prognosis of UDCA response was recently reported in a large multicenter study. Biochemical nonresponse and an APRI of $>0.54$ after 1 year treatment were associated with a 10 -year complication rate of $37.4 \%$ as compared to only $3.2 \%$ in biochemical responders with an APRI of $\leq 0.54 .{ }^{112}$

The major problem of all these studies is the post hoc analysis of UDCA response, which is usually after 1 year. A small study assessed the prediction of response to UDCA using biochemical values at 3 and 6 months and reported that biochemical responses at 6 months can be used with equal accuracy with those at 1 year. ${ }^{113}$

It would be most interesting if the prediction of UDCA response could be based on pretreatment parameters only.
A large cross-sectional study from the UK of 2353 patients suggested that the age at diagnosis was a strong predictor of UDCA response. Patients older than 70 years had a $90 \%$ response rate compared to only $50 \%$ for patients younger than $30 .{ }^{114}$

miRNAs as biomarkers to predict the response to treatment can possibly be used. A small study reported that the overexpression of miR-299-5p in PBMCs was associated with nonresponse to UDCA treatment. ${ }^{115}$

Elevated levels of serum IL-8 and sCD14 before UDCA therapy were significantly associated with both liver decompensation and liver-related death or liver transplantation. ${ }^{116}$ These findings could be a good substitute for the complex models if properly validated.

\section{Conclusion}

Although many potential biomarkers have recently been proposed, they lack proper validation. The situation is clearly presented when the few meta-analyses are scrutinized. They all start with a very large number of studies and finally analyze $<10 \%$ of them. It seems therefore that older immunological markers are still the most useful biomarkers for diagnosis and in part for the prediction of disease progression. However, models based on biochemical or hematological parameters are capable to predict response to treatment. Table 1 summarizes the main reports on biomarkers that can be used in different aspects of PBC.

\section{Author contributions}

Designed the review, revised the article and finally approved the text: EK. Acquired the data and revised the manuscript: DS. Acquired the data and prepared the draft: AV. All authors contributed toward data analysis, drafting and revising the paper and agree to be accountable for all aspects of the work.

\section{Disclosure}

The authors report no conflicts of interest in this work.

\section{References}

1. Boonstra K, Beuers U, Ponsioen CY. Epidemiology of primary sclerosing cholangitis and primary biliary cirrhosis: a systematic review. J Hepatol. 2012;56(5):1181-1188.

2. Koulentaki M, Mantaka A, Sifaki-Pistolla D, Thalassinos E, Tzanakis N, Kouroumalis E. Geoepidemiology and space-time analysis of primary biliary cirrhosis in Crete, Greece. Liver Int. 2014;34(7):e200-e207.

3. Lindor KD, Gershwin ME, Poupon R, Kaplan M, Bergasa NV, Heathcote EJ. Primary biliary cirrhosis. Hepatology. 2009;50(1): 291-308.

4. Achenza MI, Meda F, Brunetta E, Selmi C. Serum autoantibodies for the diagnosis and management of autoimmune liver diseases. Expert Rev Gastroenterol Hepatol. 2012;6(6):717-729. 
5. Yamagiwa S, Kamimura H, Takamura M, Aoyagi Y. Autoantibodies in primary biliary cirrhosis: recent progress in research on the pathogenetic and clinical significance. World J Gastroenterol. 2014;20(10):2606-2612.

6. Benson GD, Kikuchi K, Miyakawa H, Tanaka A, Watnik MR, Gershwin ME. Serial analysis of antimitochondrial antibody in patients with primary biliary cirrhosis. Clin Dev Immunol. 2004;11(2):129-133.

7. Carey EJ, Ali AH, Lindor KD. Primary biliary cirrhosis. Lancet. 2015;386(10003):1565-1575.

8. Wang J, Budamagunta MS, Voss JC, et al. Antimitochondrial antibody recognition and structural integrity of the inner lipoyl domain of the E2 subunit of pyruvate dehydrogenase complex. J Immunol. 2013;191(5):2126-2133.

9. Hua S, Zhaoa F, Wang Q, Chen WX. The accuracy of the antimitochondrial antibody and the M2 subtype test for diagnosis of primary biliary cirrhosis: a meta-analysis. Clin Chem Lab Med. 2014;52(11):1533-1542.

10. Han E, Joa SJ, Lee H, et al. Clinical relevance of combined antimitochondrial M2 detection assays for primary biliary cirrhosis. Clin Chim Acta. 2017;464:113-117.

11. Lu C, Hou X, Li M, et al. Detection of AMA-M2 in human saliva: potentials in diagnosis and monitoring of primary biliary cholangitis. Sci Rep. 2017;7(1):796.

12. Gabeta S, Norman GL, Liaskos C, et al. Diagnostic relevance and clinical significance of the new enhanced performance M2 (MIT3) ELISA for the detection of IgA and IgG antimitochondrial antibodies in primary biliary cirrhosis. J Clin Immunol. 2007;27(4):378-387.

13. Oertelt S, Rieger R, Selmi C, et al. A sensitive bead assay for antimitochondrial antibodies: chipping away at AMA-negative primary biliary cirrhosis. Hepatology. 2007;45(3):659-665.

14. Leung PSC, Choi J, Yang G, Woo E, Kenny TP, Gershwin ME. A contemporary perspective on the molecular characteristics of mitochondrial autoantigens and diagnosis in primary biliary cholangitis. Expert Rev Mol Diagn. 2016;16(6):697-705.

15. Muratori P, Muratori L, Ferrari R, et al. Characterization and clinical impact of antinuclear antibodies in primary biliary cirrhosis. $\mathrm{Am} \mathrm{J}$ Gastroenterol. 2003;98(2):431-437.

16. Duarte-Rey C, Bogdanos D, Yang CY, et al. Primary biliary cirrhosis and the nuclear pore complex. Autoimmun Rev. 2012;11(12):898-902.

17. Granito A, Muratori P, Quarneti C, Pappas G, Cicola R, Muratori L. Antinuclear antibodies as ancillary markers in primary biliary cirrhosis. Expert Rev Mol Diagn. 2012;12(1):65-74.

18. Bogdanos DP, Liaskos C, Pares A, et al. Anti-gp210 antibody mirrors disease severity in primary biliary cirrhosis. Hepatology. 2007;45(6):1583; author reply 1583-1584.

19. Sfakianaki O, Koulentaki M, Tzardi M, et al. Peri-nuclear antibodies correlate with survival in Greek primary biliary cirrhosis patients. World J Gastroenterol. 2010;16(39):4938-4943.

20. Nakamura M, Kondo H, Mori T, et al. Anti-gp210 and anti-centromere antibodies are different risk factors for the progression of primary biliary cirrhosis. Hepatology. 2007;45(1):118-127.

21. Hu SL, Zhao FR, Hu Q, Chen WX. Meta-analysis assessment of GP210 and SP100 for the diagnosis of primary biliary cirrhosis. PLoS One. 2014;9(7):e101916.

22. Bizzaro N, Covini G, Rosina F, et al. Overcoming a "probable" diagnosis in antimitochondrial antibody negative primary biliary cirrhosis: study of 100 sera and review of the literature. Clin Rev Allerg Immunol. 2012;42(3):288-297.

23. Wesierska-Gadek J, Klima A, Komina O, Ranftler C, Invernizzi P, Penner E. Characterization of autoantibodies against components of the nuclear pore complexes: high frequency of anti-p62 nucleoporin antibodies. Ann NY Acad Sci. 2007;1109:519-530.

24. Mytilinaiou MG, Meyer W, Scheper T, et al. Diagnostic and clinical utility of antibodies against the nuclear body promyelocytic leukaemia and Sp100 antigens in patients with primary biliary cirrhosis. Clin Chim Acta. 2012;413(15-16):1211-1216.
25. Granito A, Yang WH, Muratori L, et al. PML nuclear body component sp140 is a novel autoantigen in primary biliary cirrhosis. $\mathrm{Am} \mathrm{J}$ Gastroenterol. 2010;105(1):125-131.

26. Villalta D, Sorrentino MC, Girolami E, et al; Study Group on Autoimmune Diseases of the Italian Society of Laboratory Medicine. Autoantibody profiling of patients with primary biliary cirrhosis using a multiplexed line-blot assay. Clin Chim Acta. 2015;438:135-138.

27. Li YZ, Hu CJ, Leng XM, Zhao GF, Li N, Xu Y. Promising diagnostic biomarkers for primary biliary cirrhosis identified with magnetic beads and MALDI-TOF-MS. Anat Rec (Hoboken). 2009;292(3):455-460.

28. Hu CJ, Song G, Huang W, et al. Identification of new autoantigens for primary biliary cirrhosis using human proteome microarrays. $\mathrm{Mol}$ Cell Proteomics. 2012;11(9):669-680.

29. Norman GL, Yang CY, Ostendorff HP, et al. Anti-Kelch-like 12 and anti-hexokinase 1: novel autoantibodies in primary biliary cirrhosis. Liver Int. 2015;35(2):642-651.

30. de Liso F, Matinato C, Ronchi M, Maiavacca R. The diagnostic accuracy of biomarkers for diagnosis of primary biliary cholangitis (PBC) in anti-mitochondrial antibody (AMA)-negative PBC patients: a review of literature. Clin Chem Lab Med. 2017;56(1):25-31.

31. Bell LN, Wulff J, Comerford M, Vuppalanchi R, Chalasani N. Serum metabolic signatures of primary biliary cirrhosis and primary sclerosing cholangitis. Liver Int. 2015;35(1):263-274.

32. Tang YM, Wang JP, Bao WM, et al. Urine and serum metabolomic profiling reveals that bile acids and carnitine may be potential biomarkers of primary biliary cirrhosis. Int J Mol Med. 2015;36(2):377-385.

33. Lian JS, Liu W, Hao SR, et al. A serum metabolomic analysis for diagnosis and biomarker discovery of primary biliary cirrhosis and autoimmune hepatitis. Hepatobiliary Pancreat Dis Int. 2015;14(4): 13-421.

34. Hao J, Yang T, Zhou Y, et al. Serum metabolomics analysis reveals a distinct metabolic profile of patients with primary biliary cholangitis. Sci Rep. 2017;7(1):784.

35. Amathieu R, Triba MN, Goossens C, et al. Nuclear magnetic resonance based metabolomics and liver diseases: recent advances and future clinical applications. World J Gastroenterol. 2016;22(1):417-426.

36. Yu M, Zhu Y, Cong Q, Wu C. Metabonomics research progress on liver diseases. Can J Gastroenterol Hepatol. 2017;2017:8467192.

37. Pisarello MJ, Loarca L, Ivanics T, Morton L, LaRusso N. MicroRNAs in the cholangiopathies: pathogenesis, diagnosis, and treatment. J Clin Med. 2015;4(9):1688-1712.

38. Padgett KA, Lan RY, Leung PC, et al. Primary biliary cirrhosis is associated with altered hepatic microRNA expression. J Autoimmun. 2009;32(3-4):246-253.

39. Qin B, Huang F, Liang Y, Yang Z, Zhong R. Analysis of altered microRNA expression profiles in peripheral blood mononuclear cells from patients with primary biliary cirrhosis. J Gastroenterol Hepatol. 2013;28(3):543-550.

40. Qian C, Chen SX, Ren CL, Zhong RQ, Deng AM, Qin Q. Abnormal expression of miR-let-7b in primary biliary cirrhosis and its clinical significance. Zhonghua Gan Zang Bing Za Zhi. 2013;21(7):533-536.

41. Ninomiya M, Kondo Y, Funayama R, et al. Distinct microRNAs expression profile in primary biliary cirrhosis and evaluation of miR 505-3p and miR197-3p as novel biomarkers. PLoS One. 2013;8(6):e66086.

42. Katsumi T, Ninomiya M, Nishina T, et al. MiR-139-5p is associated with inflammatory regulation through c-FOS suppression, and contributes to the progression of primary biliary cholangitis. Lab Invest. 2016;96(11):1165-1177.

43. Tan Y, Pan T, Ye Y, et al. Serum microRNAs as potential biomarkers of primary biliary cirrhosis. PLoS One. 2014;9(10):e111424.

44. Liang DY, Hou YQ, Luo LJ, Ao L. Altered expression of miR-92a correlates with Th17 cell frequency in patients with primary biliary cirrhosis. Int J Mol Med. 2016;38(1):131-138.

45. Yang CY, Ma X, Tsuneyama K, et al. IL-12/Th1 and IL-23/Th17 biliary microenvironment in primary biliary cirrhosis: implications for therapy. Hepatology. 2014;59(5):1944-1953. 
46. Qian C, Jiang T, Zhang W, et al. Increased IL-23 and IL-17 expression by peripheral blood cells of patients with primary biliary cirrhosis. Cytokine. 2013;64(1):172-180.

47. Banales JM, Saez E, Uriz M, et al. Up-regulation of microRNA 506 leads to decreased $\mathrm{Cl}$-/HCO3 - anion exchanger 2 expression in biliary epithelium of patients with primary biliary cirrhosis. Hepatology. 2012;56(2):687-697.

48. Ananthanarayanan M, Banales JM, Guerra MT, et al. Posttranslational regulation of the type III inositol 1,4,5-trisphosphate receptor by miRNA-506. J Biol Chem. 2015;290(1):184-196.

49. Erice O, Munoz-Garrido P, Vaquero J, et al. MicroRNA-506 promotes primary biliary cholangitis-like features in cholangiocytes and immune activation. Hepatology. 2017;67(4):1420-1440.

50. Nakagawa R, Muroyama R, Saeki C, et al. miR-425 regulates inflammatory cytokine production in CD4+ T cells via N-Ras upregulation in primary biliary cholangitis. J Hepatol. 2017;66(6):1223-1230.

51. Wang X, Wen X, Zhou J, et al. MicroRNA-223 and microRNA-21 in peripheral blood B cells associated with progression of primary biliary cholangitis patients. PLoS One. 2017;12(9):e0184292.

52. Li L, Masica D, Ishida M, et al. Human bile contains microRNAladen extracellular vesicles that can be used for cholangiocarcinoma diagnosis. Hepatology. 2014;60(3):896-907.

53. Tomiyama T, Yang GX, Zhao M, et al. The modulation of co stimulatory molecules by circulating exosomes in primary biliary cirrhosis. Cell Mol Immunol. 2017;14(3):276-284.

54. Esparza-Baquer A, Labiano I, Bujanda L, Perugorria MJ, Banales JM. MicroRNAs in cholangiopathies: potential diagnostic and therapeutic tools. Clin Res Hepatol Gastroenterol. 2016;40(1):15-27.

55. Kennedy I, Francis H, Meng F, Glaser S, Alpini G. Diagnostic and therapeutic potentials of microRNAs in cholangiopathies. Liver Res. 2017;1(1):34-41.

56. Olaizola P, Lee-Law PY, Arbelaiz A, et al. MicroRNAs and extracellular vesicles in cholangiopathies. Biochim Biophys Acta. 2018;1864(4 pt B):1293-1307.

57. Klein R, Pointner H, Zilly W, et al. Antimitochondrial antibody profiles in primary biliary cirrhosis distinguish at early stages between a benign and a progressive course: a prospective study on 200 patients followed for 10 years. Liver. 1997;17(3):119-128.

58. Vleggaar FP, van Buuren HR. No prognostic significance of antimitochondrial antibody profile testing in primary biliary cirrhosis. Hepatogastroenterology. 2004;51(58):937-940.

59. Rigopoulou EI, Davies ET, Bogdanos DP, et al. Antimitochondrial antibodies of immunoglobulin G3 subclass are associated with a more severe disease course in primary biliary cirrhosis. Liver Int. 2007;27(9):1226-1231.

60. Wesierska-Gadek J, Penner E, Battezzati PM, et al. Correlation of initial autoantibody profile and clinical outcome in primary biliary cirrhosis. Hepatology. 2006;43(5):1135-1144.

61. Nakamura M, Shimizu-Yoshida Y, Takii Y, et al. Antibody titer to gp210-C terminal peptide as a clinical parameter for monitoring primary biliary cirrhosis. J Hepatol. 2005;42(3):386-392.

62. Mandai S, Kanda E, Arai Y, et al. Anti-centromere antibody is an independent risk factor for chronic kidney disease in patients with primary biliary cirrhosis. Clin Exp Nephrol. 2013;17(3):405-410.

63. Liberal R, Grant CR, Sakkas L, Bizzaro N, Bogdanos DP. Diagnostic and clinical significance of anti-centromere antibodies in primary biliary cirrhosis. Clin Res Hepatol Gastroenterol. 2013;37(6):572-585.

64. Miyachi K, Hankins RW, Matsushima H, et al. Profile and clinical significance of anti-nuclear envelope antibodies found in patients with primary biliary cirrhosis: a multicenter study. J Autoimmun. 2003;20(3):247-254.

65. Tana MM, Shums Z, Milo J, et al. The significance of autoantibody changes over time in primary biliary cirrhosis. Am J Clin Pathol. 2015;144(4):601-606.

66. Moll R, Divo M, Langbein L. The human keratins: biology and pathology. Histochem Cell Biol. 2008;129(6):705-733.
67. Tao GZ, Li DH, Zhou Q, et al. Monitoring of epithelial cell caspase activation via detection of durable keratin fragment formation. JPathol. 2008;215(2):164-174.

68. Denk G, Omary AJ, Reiter FP, et al. Soluble intracellular adhesion molecule, M30 and M65 as serum markers of disease activity and prognosis in cholestatic liver diseases. Hepatol Res. 2014;44(13):1286-1298.

69. Sekiguchi T, Umemura T, Fujimori N, et al. Serum cell death biomarkers for prediction of liver fibrosis and poor prognosis in primary biliary cirrhosis. PLoS One. 2015;10(6):e0131658.

70. Miyachi K, Hosaka H, Nakamura N, et al. Anti-p97/VCP antibodies: an autoantibody marker for a subset of primary biliary cirrhosis patients with milder disease? Scand J Immunol. 2006;63(5):376-382.

71. Wunsch E, Krawczyk M, Milkiewicz M, et al. Serum autotaxin is a marker of the severity of liver injury and overall survival in patients with cholestatic liver diseases. Sci Rep. 2016;6:30847.

72. Wunsch E, Milkiewicz M, Wasik U, et al. Expression of hepatic fibroblast growth factor 19 is enhanced in primary biliary cirrhosis and correlates with severity of the disease. Sci Rep. 2015;5:13462.

73. Zhao P, Liu WW, Li JF, et al. Predictors of liver failure in primary biliary cirrhosis. Ups J Med Sci. 2015;120(1):47-51.

74. Harada K, Kakuda Y, Nakamura M, Shimoda S, Nakanuma Y. Clinicopathological significance of serum fractalkine in primary biliary cirrhosis. Dig Dis Sci. 2013;58(10):3037-3043.

75. Norman GL, Gatselis NK, Shums Z, et al. Cartilage oligomeric matrix protein: a novel non-invasive marker for assessing cirrhosis and risk of hepatocellular carcinoma. World J Hepatol. 2015;7(14): 1875-1883.

76. Deng $\mathrm{C}, \mathrm{Hu} \mathrm{C}$, Wang $\mathrm{L}$, et al. Serological comparative proteomics analysis of mitochondrial autoantibody-negative and -positive primary biliary cirrhosis. Electrophoresis. 2015;36:1588-1595.

77. Umemura T, Joshita S, Sekiguchi T, et al. Serum Wisteria floribunda agglutinin-positive Mac-2-binding protein level predicts liver fibrosis and prognosis in primary biliary cirrhosis. Am J Gastroenterol. 2015;110(6):857-864

78. Trivedi PJ, Bruns T, Cheung A, et al. Optimising risk stratification in primary biliary cirrhosis: AST/platelet ratio index predicts outcome independent of ursodeoxycholic acid response. $J$ Hepatol. 2014;60(6):1249-1258.

79. Joshita S, Umemura T, Ota M, Tanaka E. AST/platelet ratio index associates with progression to hepatic failure and correlates with histological fibrosis stage in Japanese patients with primary biliary cirrhosis. J Hepatol. 2014;61(6):1443-1445.

80. Cheung KS, Seto WK, Fung J, Lai CL, Yuen MF. Prognostic factors for transplant-free survival and validation of prognostic models in Chinese patients with primary biliary cholangitis receiving ursodeoxycholic acid. Clin Transl Gastroenterol. 2017;8(6):e100.

81. Weinmann A, Sattler T, Unold HP, et al. Predictive scores in primary biliary cirrhosis: a retrospective single center analysis of 204 patients. J Clin Gastroenterol. 2015;49(5):438-447.

82. Ali AH, Sinakos E, Silveira MG, Jorgensen RA, Angulo P, Lindor KD. Varices in early histological stage primary biliary cirrhosis. $J$ Clin Gastroenterol. 2011;45(7):e66-e71.

83. Voumvouraki A, Koulentaki M, Notas G, Sfakianaki O, Kouroumalis E. Serum surrogate markers of liver fibrosis in primary biliary cirrhosis. Eur J Intern Med. 2011;22(1):77-83.

84. Zhang HC, Hu RF, Zhu T, Tong L, Zhang QQ. Primary biliary cirrhosis degree assessment by acoustic radiation force impulse imaging and hepatic fibrosis indicators. World $J$ Gastroenterol. 2016;22(22):5276-5284.

85. Tahtaci M, Yurekli OT, Bolat AD, et al. Increased mean platelet volume is related to histologic severity of primary biliary cirrhosis. Eur J Gastroenterol Hepatol. 2015;27(12):1382-1385.

86. Nishikawa H, Enomoto H, Iwata Y, et al. Impact of serum Wisteria floribunda agglutinin positive Mac-2-binding protein and serum interferon-c-inducible protein-10 in primary biliary cirrhosis. Hepatol Res. 2016;46(6):575-583. 
87. Haga Y, Kanda T, Sasaki R, Nakamura M, Takahashi K, Wu S. Serum Wisteria floribunda agglutinin-positive Mac-2 binding protein could not always predict early cirrhosis in non-viral liver diseases. Diseases. 2016;4(4):E38.

88. Shirabe K, Bekki Y, Gantumur D, et al. Mac-2 binding protein glycan isomer (M2BPGi) is a new serum biomarker for assessing liver fibrosis: more than a biomarker of liver fibrosis. J Gastroenterol. Epub 2018 Jan 9.

89. Deng H, Qi X, Guo X. Diagnostic accuracy of APRI, AAR, FIB-4, FI, king, Lok, Forns, and FibroIndex scores in predicting the presence of esophageal varices in liver cirrhosis: a systematic review and metaanalysis. Medicine (Baltimore). 2015;94(42):e1795.

90. Nyblom H, Björnsson E, Simrén M, Aldenborg F, Almer S, Olsson R. The AST/ALT ratio as an indicator of cirrhosis in patients with PBC Liver Int. 2006;26(7):840-845.

91. Alempijevic T, Krstic M, Jesic R, et al. Biochemical markers for noninvasive assessment of disease stage in patients with primary biliary cirrhosis. World J Gastroenterol. 2009;15(5):591-594.

92. Floreani A, Cazzagon N, Martines D, Cavalletto L, Baldo V, Chemello L. Performance and utility of transient elastography and noninvasive markers of liver fibrosis in primary biliary cirrhosis. Dig Liver Dis. 2011;43(11):887-892.

93. Stasi C, Leoncini L, Biagini MR, et al. Assessment of liver fibrosis in primary biliary cholangitis: comparison between indirect serum markers and fibrosis morphometry. Dig Liver Dis. 2016;48(3):298-301.

94. Olmez S, Sayar S, Avcioglu U, et al. The relationship between liver histology and noninvasive markers in primary biliary cirrhosis. Eur J Gastroenterol Hepatol. 2016;28(7):773-776.

95. Mayo MJ, Parkes J, Adams-Huet B, et al. Prediction of clinical outcomes in primary biliary cirrhosis by serum enhanced liver fibrosis assay. Hepatology. 2008;48(5):1549-1557.

96. Xie Q, Zhou X, Huang P, Wei J, Wang W, Zheng S. The performance of enhanced liver fibrosis (ELF) test for the staging of liver fibrosis: a meta-analysis. PLoS One. 2014;9(4):e92772.

97. Wang $\mathrm{H}, \mathrm{Xu} \mathrm{H}$, Wang $\mathrm{X}$, et al. Red blood cell distribution width to platelet ratio is related to histologic severity of primary biliary cirrhosis. Medicine (Baltimore). 2016;95(11):e3114.

98. Wang Z, Liu X, Xu H, Qu L, Zhang D, Gao P. Platelet count to spleen thickness ratio is related to histologic severity of primary biliary cholangitis. Medicine (Baltimore). 2018;97(7):e9843.

99. Swets JA. Measuring the accuracy of diagnostic systems. Science. 1988;240(4857):1285-1293.

100. Mendes LC, Stucchi RS, Vigani AG. Diagnosis and staging of fibrosis in patients with chronic hepatitis $\mathrm{C}$ : comparison and critical overview of current strategies. Hepat Med. 2018;10:13-22.

101. Saffioti F, Gurusamy KS, Eusebi LH, Tsochatzis E, Davidson BR, Thorburn D. Pharmacological interventions for primary biliary cholangitis: an attempted network meta-analysis. Cochrane Database Syst Rev. 2017;3:CD011648.

102. Gatselis NK, Zachou K, Norman GL, et al. Clinical significance of the fluctuation of primary biliary cirrhosis-related autoantibodies during the course of the disease. Autoimmunity. 2013;46(7):471-479.
103. Agmon-Levin N, Kopilov R, Selmi C, et al. Vitamin D in primary biliary cirrhosis, a plausible marker of advanced disease. Immunol Res. 2015;61(1-2):141-146.

104. Guo GY, Shi YQ, Wang L, et al. Serum vitamin D level is associated with disease severity and response to ursodeoxycholic acid in primary biliary cirrhosis. Aliment Pharmacol Ther. 2015;42(2):221-230.

105. Pares A, Caballeria L, Rodes J. Excellent long-term survival in patients with primary biliary cirrhosis and biochemical response to ursodeoxycholic acid. Gastroenterology. 2006;130(3):715-720.

106. Corpechot C, Abenavoli L, Rabahi N, et al. Biochemical response to ursodeoxycholic acid and long-term prognosis in primary biliary cirrhosis. Hepatology. 2008;48(3):871-877.

107. Kumagi T, Guindi M, Fischer SE, et al. Baseline ductopenia and treatment response predict long-term histological progression in primary biliary cirrhosis. Am J Gastroenterol. 2010;105(10):2186-2194.

108. Corpechot C, Chazouilleres O, Poupon R. Early primary biliary cirrhosis: biochemical response to treatment and prediction of long-term outcome. J Hepatol. 2011;55(6):1361-1367.

109. Papastergiou V, Tsochatzis EA, Rodriguez-Peralvarez M, et al. Biochemical criteria at 1 year are not robust indicators of response to ursodeoxycholic acid in early primary biliary cirrhosis: results from a 29-year cohort study. Aliment Pharmacol Ther. 2013;38(11-12): 1354-1364.

110. Lammers WJ, van Buuren HR, Hirschfield GM, et al; Global PBC Study Group. Levels of alkaline phosphatase and bilirubin are surrogate end points of outcomes of patients with primary biliary cirrhosis: an international follow-up study. Gastroenterology. 2014;147(6):13381349.e5; quiz e15.

111. Lammers WJ, Hirschfield GM, Corpechot C, et al; Global PBC Study Group. Development and validation of a scoring system to predict outcomes of patients with primary biliary cirrhosis receiving ursodeoxycholic acid therapy. Gastroenterology. 2015;149(7): 1804-1812.

112. Harms MH, Lammers WJ, Thorburn D, et al. Major hepatic complications in ursodeoxycholic acid-treated patients with primary biliary cholangitis: risk factors and time trends in incidence and outcome. Am J Gastroenterol. 2018;113(2):254-264.

113. Zhang LN, Shi TY, Shi XH, et al. Early biochemical response to ursodeoxycholic acid and long-term prognosis of primary biliary cirrhosis: results of a 14-year cohort study. Hepatology. 2013;58(1): 264-272.

114. Carbone M, Mells GF, Pells G, et al; UK PBC Consortium. Sex and age are determinants of the clinical phenotype of primary biliary cirrhosis and response to ursodeoxycholic acid. Gastroenterology. 2013;144(3) $560-569$.

115. Katsushima F, Takahashi A, Sakamoto N, Kanno Y, Abe K, Ohira H. Expression of microRNAs in peripheral blood mononuclear cells from primary biliary cirrhosis patients. Hepatol Res. 2014;44(10):E189-E197.

116. Umemura T, Sekiguchi T, Joshita S, et al. Association between serum soluble CD14 and IL-8 levels and clinical outcome in primary biliary cholangitis. Liver Int. 2017;37(6):897-905.

\section{Hepatic Medicine: Evidence and Research}

\section{Publish your work in this journal}

Hepatic Medicine: Evidence and Research is an international, peerreviewed, open access journal covering all aspects of adult and pediatric hepatology in the clinic and laboratory including the following topics: Pathology, pathophysiology of hepatic disease; Investigation and treatment of hepatic disease; Pharmacology of drugs used for the treatment of hepatic disease. Issues of patient safety and quality of care will also be considered. The manuscript management system is completely online and includes a very quick and fair peer-review system, which is all easy to use. Visit http://www.dovepress.com/testimonials.php to read real quotes from published authors. 